\title{
Erratum to: Knockdown of HMGN5 suppresses the viability and invasion of human urothelial bladder cancer 5637 cells in vitro and in vivo
}

\author{
Yu Gan ${ }^{1} \cdot$ Jing $\operatorname{Tan}^{1} \cdot$ Jianfu Yang ${ }^{1} \cdot$ Yihong Zhou $^{1} \cdot$

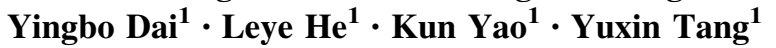

Published online: 21 April 2015

(C) Springer Science+Business Media New York 2015

\section{Erratum to: Med Oncol (2015) 32:136 DOI 10.1007/s12032-015-0594-y}

The following errors were inadvertently omitted by the author in the original publication, and it has been corrected with this erratum.

1. "Western blot also demonstrated that the expression of E-cadherin and VEGF-C was decreased in 5637 cells depleted of HMGN5." should be replaced by "Western blot also demonstrated that the expression of E-cadherin was enhanced, while the expression of VEGF-C was decreased in 5637 cells depleted of HMGN5." in the 23-25th line of the first column under the Abstract section on page 1 .

2. In the legend of Fig. 3 on page 6, "HMGN5 knockdown inhibited the expression of E-cadherin $(p<$ $0.01)$ and VEGF-C $(p<0.01)$ in 5637 cells." should be replaced by "HMGN5 knockdown enhanced the expression of E-cadherin $(p<0.01)$, but inhibited the expression of VEGF-C $(p<0.01)$ in 5637 cells."

3. "This study aimed to further investigate the oncogenic role of HMGN5 in UBC 5637 cells employing in vitro and in vivo models and explored the mechanism." should be replaced by "This study aimed to further investigate the oncogenic role of HMGN5 in UBC 5637 cells employing in vitro and in vivo models and to explore the mechanism." In the 6-9th line of the first column under the Abstract section on page 1.

4. "HMGN5 knockdown suppressed invasion induced G1/S cell cycle arrest but not apoptosis and thus contributed to decreased cell viability in UBC 5637 cells." should be replaced by "HMGN5 knockdown suppressed invasion, and induced G1/S cell cycle arrest but not apoptosis and thus contributed to decreased cell viability in UBC 5637 cells." In the 17-20th line of the first column under the Abstract section on page 1.

The online version of the original article can be found under doi:10.1007/s12032-015-0594-y.

\section{Kun Yao}

yaokun52@163.com

1 Department of Urology, The Third Xiangya Hospital of Central South University, 138 Tongzipo Road, Changsha, Hunan 410013, People's Republic of China 